\title{
X\#Ray Line Diagnostics of Hot Accretion Flows around Black Holes
}

\section{Citation}

Perna, Rosalba, John Raymond, and Ramesh Narayan. 2000. "X\#Ray Line Diagnostics of Hot Accretion Flows around Black Holes." The Astrophysical Journal 541 (2): 898-907. https:// doi.org/10.1086/309466.

\section{Permanent link}

http://nrs.harvard.edu/urn-3:HUL.InstRepos:41384932

\section{Terms of Use}

This article was downloaded from Harvard University's DASH repository, and is made available under the terms and conditions applicable to Other Posted Material, as set forth at http:// nrs.harvard.edu/urn-3:HUL.InstRepos:dash.current.terms-of-use\#LAA

\section{Share Your Story}

The Harvard community has made this article openly available.

Please share how this access benefits you. Submit a story.

Accessibility 


\title{
$X$-ray Line Diagnostics of Hot Accretion Flows around Black Holes
}

\author{
Rosalba Perna ${ }^{1}$, John Raymond and Ramesh Narayan \\ Harvard-Smithsonian Center for Astrophysics, 60 Garden Street, Cambridge, MA 02138
}

\begin{abstract}
We compute $X$-ray emission lines from thermal plasma in hot accretion flows. We show that line profiles are strong probes of the gas dynamics, and we present line-ratio diagnostics which are sensitive to the distribution of mass with temperature in the flow. We show how these can be used to constrain the run of density with radius, and the size of the hot region. We also present diagnostics which are primarily sensitive to the importance of recombination versus collisional ionization, and which could help discriminate ADAFs from photoionization-dominated accretion disk coronae. We apply our results to the Galactic center source Sagittarius $A^{*}$ and to the nucleus of M87. We find that the brightest predicted lines are within the detection capability of current $X$-ray instruments.
\end{abstract}

Subject headings: accretion, accretion disks — black hole physics — galaxies: nuclei — Galaxy: center - radiation mechanisms: thermal $-X$-rays: stars

\section{Introduction}

Many $X$-ray sources in the sky, such as stellar mass black holes in $X$-ray binaries, and supermassive black holes in the nucleus of our Galaxy and in other galaxies, are believed to be powered by gas falling into the black hole. In the past few years, advection-dominated accretion flows (ADAFs) have been successfully used to model both spectra and luminosities in some of these systems (Narayan \& Yi 1994, 1995; Abramowicz et al. 1995; see Narayan, Mahadevan \& Quataert 1998 and Kato, Fukue, \& Mineshige 1998 for reviews). A feature of these models is that the gas is very hot, approaching the virial temperature at each radius, and optically thin. The emission from the inner region of the flow is dominated by synchrotron radiation and Comptonization, and it has been studied in detail by a number of authors (see Liang 1998 and Mushotzky, Done, \& Pounds 1993 for reviews of the continuum spectral energy distribution for these processes, and Narayan et al. 1998 for application to ADAFs). On the other hand, the outer region of the flow, with temperatures around $10^{7}-10^{8} \mathrm{~K}$, is dominated by bremsstrahlung and thermal $X$-ray line emission from hot, optically thin gas.

\footnotetext{
${ }^{1}$ Harvard Society of Fellows
} 
The electron temperature in an ADAF varies roughly as $T_{e} \sim 10^{12} \mathrm{~K} / r$, for $r \gtrsim 10^{3}$ (Narayan \& Yi 1995b), where $r$ is the radius in Schwarzschild units $\left[2.95 \times 10^{5}\left(M / M_{\odot}\right) \mathrm{cm}\right.$, where $M$ is the mass of the black hole]. Thus, the region of the flow between $r \sim 10^{4}$ and $r \sim 10^{5}$ is the most important for thermal line emission. Narayan \& Raymond (1999) pointed out significant diagnostic possibilities that the emission from this region has. In particular, they studied equivalent widths of emission lines, and showed how these differ in various types of ADAF models. Spectral fits to the emission from some accreting systems have required the assumption that some mass is lost to winds during accretion (e.g. Di Matteo et al. 1999). The modification is described by a parameter $p$ (defined in $\S 2$ below), which leads to a modified density profile as a function of radius. Another possibility is that the ADAF may be convective, in which case the density profile is again strongly modified (Narayan, Igumenshchev \& Abramovicz 2000, Quataert \& Gruzinov 2000). In effect, this density profile is similar to that for an ADAF with a $p=1$ wind. These possibilities lead to some uncertainties in the details of ADAF models. Another uncertainty is related to the size of the ADAF region. In some systems (such as V404 Cyg, M87), the ADAF is believed to extend from the black hole horizon to a transition radius $r_{\text {tr }}$, beyond which the flow consists of a thin disk plus a corona. But in other systems, e.g. the Galactic center source Sagittarius $\mathrm{A}^{*}\left(\mathrm{Sgr} \mathrm{A}^{*}\right)$, there is no evidence for an outer thin disk. Narayan \& Raymond did not study the effect of varying $r_{\operatorname{tr}}$ on the lines. Moreover, they assumed equilibrium values for the ionic concentrations of the elements, and adopted height-averaged equations for the ADAF. We improve on these aspects of their model by computing time-dependent ionizations fractions, and also adopting the general ADAF solution for the motion of the flow in the $r \theta$ plane.

In this paper we present several diagnostics provided by thermal emission from the hot plasma in the outer region of the flow. Taken together, these can set strong constraints on important properties of the flow itself. We apply our results to Sgr A* and to the nucleus of M87.

In $\S 3.1$, we calculate line profiles. In ADAF models, these are independent of the mass of the central black hole. However, they depend on the temperature of the gas (or actually on how much gas there is within each temperature range), on the amount of rotation of the gas and on the inclination angle (i.e. the angle between the axis of rotation of the flow and the direction of observation). We show that there are several other diagnostics that constrain some of these parameters independently $(\S 3.2)$, such as line ratios and the slope of the continuum. Relative intensities of lines from low and high ionization states are primarily sensitive to the relative density at large and small radii. In ADAF models, the run of gas density with radius is uncertain, as is the position of the transition radius (if there is one) to a thin disk plus a corona. Some line ratios are very sensitive to these aspects of the flow, and they can thus be used to set constraints on them. This is particularly useful for the case of $\operatorname{SgrA}^{*}$, where both models with and without a wind are consistent with the data (but require different assumptions for the microscopic parameters of the flow; see, e.g., Quataert \& Narayan 1999), and there is no evidence of an outer disk. In this case, we show that relative line intensities can be a useful, direct probe of the amount of mass lost to a wind during the process of accretion. Furthermore, we present other line-ratio diagnostics 
which are primarily sensitive to the importance of recombination versus collisional ionization, and we discuss how these can help discriminate ADAFs versus photoionization-dominated accretion models.

In the case of M87, spectral data are much better fitted by an ADAF with a wind (Di Matteo et al 1999). However, an uncertainty in the model for this source is the location of the transition radius $r_{\text {tr }}$ between the ADAF and an outer disk + corona. We show that line ratios are powerful diagnostics of this parameter. Moreover, for this object there is an observational estimate of the inclination angle of the disk. We then show that line profiles can be used to constrain the amount of rotation of the flow and, hence, (see §2.1) learn about some of its underlying microphysics.

\section{Model}

\subsection{Dynamics of the accretion flow}

We consider an axisymmetric ADAF model in spherical coordinates $r, \theta, \phi$. In the equatorial plane $(\theta=\pi / 2)$ we assume that the velocity components $v_{r}, v_{\phi}$ and the sound speed $c_{s}$ take the values given by the analytic self-similar solution derived by Narayan \& Yi (1994). For the variation of the velocities with $\theta$, we make use of the work of Narayan \& Yi (1995a), who have given analytic approximations for $v_{r}, v_{\phi}$ and $c_{s}$, based on exact numerical calculations. In their solution $v_{\theta}=0$, which we also assume. We thus have

$$
\begin{gathered}
v_{r}(r, \theta)=-\frac{3 \alpha}{5+2 \epsilon} r \Omega_{\mathrm{K}}(r) \sin ^{2} \theta, \\
v_{\theta}=0, \\
v_{\phi}(r, \theta)=\left(\frac{2 \epsilon}{5+2 \epsilon}\right)^{1 / 2} r \Omega_{\mathrm{K}}(r) \sin \theta, \\
c_{s}(r, \theta)=r \Omega_{\mathrm{K}}(r)\left[c_{1}-c_{2} \sin ^{2} \theta\right]^{1 / 2},
\end{gathered}
$$

where $c_{1}=2 / 5, c_{2}=(2 / 5)-2 /(5+2 \epsilon)$, and $\Omega_{\mathrm{K}}(r)=\left(G M / r^{3}\right)^{1 / 2}$ is the keplerian angular velocity at radius $r$. We express $\mathrm{r}$ in Schwarzschild units, $R_{s}=2.95 \times 10^{5}\left(M / M_{\odot}\right) \mathrm{cm}$, where $M$ is the mass of the black hole. Finally, the density is given by

$$
\rho(r, \theta)=r^{-3 / 2} \rho_{0}\left[c_{s}^{2}(\theta)\right]^{-2.25},
$$

as derived in Appendix A.

The parameters $\alpha$ and $\epsilon$ contain information on the microphysics of the flow: $\alpha$ is the viscosity parameter, for which we assume a typical value for ADAFs of $\alpha=0.1$. The parameter $\epsilon$ is related to the ratio of specific heats of the gas (Narayan \& Yi 1994). At a macroscopic level, it describes the amount of rotation of the flow. In most of the calculations we adopt $\epsilon=0.1$, but we also show how our results vary with $\epsilon$. 
Given the radial scaling of $v_{r}$, the scaling of $\rho$ is uniquely determined by the mass accretion rate $\dot{M}$ [Equation (A6)]. In the simplest scenario, $\dot{M}$ is constant throughout the flow. However, as explained in the introduction, fitting the ADAF model to the spectra of some systems requires that a fraction of the mass be lost to winds while accreting. This implies an $\dot{M}$ which decreases inward. Following the suggestion of Blandford \& Begelman (1999) and Di Matteo et al. (1999), Quataert \& Narayan (1999) studied ADAF models with winds; they assumed $\dot{M} \propto r^{p}$, and found that values of the wind parameter $p$ between 0 and 0.5 are consistent with the spectral data in several low-luminosity black holes. We consider this range of $p$ in our models.

Another uncertainty of the models is the radial extent of the ADAF. As already mentioned in $\S 1$, in some systems the ADAF region is likely to extend up to a transition radius $r_{\text {tr }}$, beyond which the flow consists of a thin disk plus a corona. Following Esin, McClintock \& Narayan (1997), in the coronal regions of these sources we set $\dot{M} \propto r^{-1}$ (a somewhat arbitrary choice).

To summarize, we adopt the following parameterization of $\dot{M}$ :

$$
\dot{M}=\dot{m}_{0} \dot{M}_{\text {Edd }}\left\{\begin{array}{ll}
\left(\frac{r}{r_{\mathrm{tr}}}\right)^{p} & \text { if } r \leq r_{\mathrm{tr}} \\
\left(\frac{r}{r_{\mathrm{tr}}}\right)^{-1} & \text { if } r \geq r_{\mathrm{tr}}
\end{array},\right.
$$

where $\dot{M}_{\text {Edd }}$ is the Eddington accretion rate. For a black hole of mass $M$, we have $\dot{M}_{\text {Edd }}=1.4 \times 10^{18}\left(M / M_{\odot}\right) \mathrm{g} \mathrm{s}^{-1}$. The particular value of the constant $\dot{m}_{0}$ in any given source is obtained by fitting the predicted flux for a given $\dot{M}$ to the observed $X$-ray spectrum. In Sgr $A^{*}$, we computed models with $p=(0,0.1,0.2,0.3,0.4,0.5)$, which gave $\dot{m}_{0}=(0.000057,0.000077,0.00016,0.00017,0.00022,0.00026)$. In M87, for the same parameters, we found $\dot{m}_{0}=(0.00086,0.0012,0.0018,0.0032,0.0061,0.01)$. It should be noted, however, that these estimates for the accretion rate are still rather uncertain. In the case of Sgr $\mathrm{A}^{*}$ the $X$-ray emission from the accretion flow is not well constrained, both because of the poor angular resolution of current observations and the large uncertainty in the absorbing column to the source. Some of the $X$-ray flux might be due to diffuse gas surrounding the source. For M87, the uncertainty in the size of the ADAF region makes it unclear whether the observed $X$-ray emission actually comes from the accretion flow onto the central black hole (Reynolds et al. 1996), or from extraneous point sources, or from the cooling flow which feeds the accretion (Quataert \& Narayan 2000)2. At any event, we also need to emphasize that, while predictions for the absolute luminosity of the lines rely on the assumed value for $\dot{m}_{0}$, our results for the relative line luminosities and for line profiles are unaffected by this uncertaintyf?.

\footnotetext{
${ }^{2}$ Observations with the ACIS instrument on CXO should be able to eliminate these sources of confusion. In fact, the resolution of this instrument is 1" which, for both $\operatorname{Sgr} \mathrm{A}^{*}$ and M87, corresponds to $r \sim 10^{5}$, that is the typical size of the ADAF region.

${ }^{3}$ Note that relative line intensities are also independent of the viscosity parameter $\alpha$ and only very weakly dependent on $\epsilon$.
} 
A non-zero value of $p$ means that there is mass outflow. In the model developed by Quataert \& Narayan, this is accounted for by taking an $\dot{M}$ which decreases with radius [cfr. Eq. (6)]. However, the motion of the flow is assumed to remain the same as for $p=0$, namely an axially-symmetric rotation with purely radial infall, as represented by Equations (11) - (3). The more general case, in which the outflow also affects the dynamics, has been studied with numerical simulations (Igumenshchev 1999; Igumenshchev \& Abramowicz 1999, 2000), but no analytical solution exists yet. In order to estimate the possible effects of an outflow on the line profiles, we have developed an approximate model of the dynamics of an ADAF with an outflow. This model leaves the rotational velocity $v_{\phi}(r, \theta)$ the same as in the no-outflow model, but introduces a $\theta$ component for the velocity, $v_{\theta}(r, \theta)$, while allowing $v_{r}(r, \theta)$ to be directed both inwards and outwards. The details of this model are described in Appendix B.

\section{2. $\quad X$-ray spectra and line profiles}

Following Narayan \& Raymond (1999), we divide the flow into two regions separated at a radius $r_{\text {in }}=10^{2}$ in Schwarzschild units. In the inner part, the emission is dominated by synchrotron, bremsstrahlung and Comptonization, and these are all computed. In this region, line emission is neglected, since the temperature is higher than $10^{9} \mathrm{~K}$ and therefore the astrophysically abundant elements are fully ionized. In the outer part, on the other hand, synchrotron emission and Comptonization are neglected, as these processes are steep functions of the temperature. In this region thermal bremsstrahlung and lines dominate the emission, and we compute them in detail. For the outer radius, we adopt $r_{\text {out }}=10^{5}$ for all the models. This is consistent with the evidence, in Sgr A*, that the stars whose winds supply most of the accreting mass are located at $r \gtrsim$ few time $10^{5}$ (Coker \& Melia 1997; Quataert, Narayan, \& Reid 1999).

We divide our flow into a grid in the $r \theta$ plane. At every point on the grid we compute velocities and densities of the ADAF model as described in the previous section. The local temperature is determined through the relation

$$
T(r, \theta)=\frac{\mu m_{p}}{k_{\mathrm{B}}} c_{s}(r, \theta)
$$

where $\mu$ is the mean molecular weight of the gas (for which we assume standard abundances), $m_{p}$ the proton mass, and $k_{\mathrm{B}}$ the Boltzmann constant. The $X$-ray spectrum in the outer region is calculated using an extended version of the Raymond \& Smith (1977) code. This computes bremsstrahlung, recombination, two-photon continua and the emission in spectral lines. Given the temperatures in the flow, most of the line emission comes from H-like and He-like ions. Included in the code are collisional excitation (see, e.g. Pradhan, Norcross \& Hummer 1981 and Pradhan 1985), recombination to excited levels of H-like and He-like ions (see, e.g. Mewe, Schrijver, \& Sylwester 1980) and dielectronic recombination satellite lines (see, e.g. Dubau et al. 1981 and Bely-Daubau et al. 1982).

Narayan \& Raymond showed that neither photoionization nor photoabsorption is very 
important, and therefore we do not consider them here. However, they also assumed ionization equilibrium, which is somewhat marginal. We have removed this restriction in our model. For each angle $\theta_{i}$ of the grid, the computation of the ionization fractions of each element proceeds from the outermost to the innermost shell at $r_{\text {in }}$, with each parcel of gas being followed while moving from a position $r_{i}$ to a position $r_{i-1}$ with velocity $v_{r}\left(r_{i}, \theta_{i}\right)$. The initial condition for the parcels of gas that start their inflow from the outermost radii is chosen to be that of ionization equilibrium at the local temperature. The subsequent behavior of the gas is not particularly sensitive to this choice. If the gas were to start at a colder temperature, it would rapidly approach the same state, due to the very fast ionization rates.

Figure 1 shows how significant the departures from equilibrium are for the flows in $\operatorname{Sgr}^{*}$ and M87. Here we compare the equilibrium (dashed line) and non-equilibrium (solid line) ionization fractions of the most important ions of iron. We see that the departure from equilibrium is only marginal. The overall tendency is a shift in the concentration peak of an ion inward by $\log \Delta r \approx 0.05$. A comparison between ionization times and infall times shows that a large departure from equilibrium is indeed not expected. For example, the ionization rate of Fe XXV is $C_{i} \approx 5 \times 10^{-13} \mathrm{~cm}^{3} \mathrm{sec}^{-1}$ (e.g. Arnaud \& Raymond 1992) at a temperature of $\sim 5 \times 10^{7} \mathrm{~K}$, where this ion is most abundant. In the case of Sgr $A^{*}$, for a density $n_{e} \sim 5 \times 10^{3} \mathrm{~cm}^{-3}$ and velocity $v_{r} \sim 100 \mathrm{~km} \mathrm{sec}^{-1}$ in the corresponding part of the flow 1 , we get an ionization time $t_{\text {ion }}=1 / n_{e} C_{i} \sim 4 \times 10^{8} \mathrm{sec}$, smaller than the infall time $t_{\text {inf }} \sim r / v_{r} \sim 7 \times 10^{9}$ sec. In the case of M87, the lower density makes $t_{\text {ion }}$ about two orders of magnitude larger, but this is more than compensated by the increase of about three orders of magnitude in the infall time (due to the larger $\left.M_{\mathrm{BH}}\right)$.

Finally, we discuss the computation of line profiles. Let $\vec{n}$ be the unit vector identifying the direction of an observer with respect to the axis of rotation of the flow. The profile of a given line that the observer will measure is then given by

$$
\Phi(v)=\int_{0}^{2 \pi} d \phi \int_{0}^{\pi} d \theta \sin \theta \int_{r_{\mathrm{in}}}^{r_{\mathrm{out}}} d r r^{2} E_{m}(r, \theta) \Phi^{\prime}(v ; r, \theta, \phi) \quad \operatorname{ergs~s}^{-1}\left(\mathrm{~km} \mathrm{~s}^{-1}\right)^{-1},
$$

where $E_{m}(r, \theta)$ is the emissivity in the line under consideration and

$$
\Phi^{\prime}(v ; r, \theta, \phi)=\frac{1}{\sqrt{\pi} \Delta} \exp \left\{-\frac{\left[v-v_{\mathrm{los}}(r, \theta, \phi)\right]^{2}}{\Delta^{2}}\right\} .
$$

Here $\Delta=\sqrt{2 k_{\mathrm{B}} T / m_{a}}$ is the thermal width of an atom of mass $m_{a}$, and $v_{\text {los }}=\vec{v} \cdot \vec{n}$ is the component of the velocity along the line of sight to the observer. If $\vec{n}=\sin i \hat{x}+\cos i \hat{z}(\hat{x}$ and $\hat{z}$ are unit vectors in cartesian coordinates), then for the case with no outflow (i.e. $v_{\theta}=0$ ), it is easy

\footnotetext{
${ }^{4}$ This is around $r \sim$ few time $10^{4}$. In the inner part of the flow, the increase in the velocity - which would cause a larger departure from equilibrium — is compensated by an increase in both density and temperature, which acts in the opposite direction.
} 
to see that

$$
v_{\mathrm{los}}(r, \theta, \phi)=v_{r}(r, \theta) \sin \theta \cos \phi \sin i+v_{r}(r, \theta) \cos \theta \cos i-v_{\phi}(r, \theta) \sin i \sin \phi
$$

The more general case with $v_{\theta} \neq 0$ is treated in Appendix B.

\section{Results}

\subsection{Line profiles}

In Sgr $\mathrm{A}^{*}$, there is no observational constraint on the inclination angle $i$ between the line of sight and the axis of rotation of the accreting gas. Figure 2 shows the profile of a strong line of iron for different choices of $i$. Note that the shape of the line is quite sensitive to $i$.

For a proper interpretation of the dominant effects in determining line broadening, we need to make some numerical estimates. First, thermal broadening. Given the range of temperatures in the flow $\left(\sim 10^{7}-10^{9} \mathrm{~K}\right)$, the resulting thermal widths are on the order of $100-150 \mathrm{~km} \mathrm{~s}^{-1}$ for lines of ions mostly abundant in the outer regions (such as O VIII, Si XIII), and on the order of 180-200 $\mathrm{km} \mathrm{s}^{-1}$ for ions produced in the inner region, such as Fe XXVI. Thermal widths must then be compared with the velocities due to the bulk motion of the gas. Typical radial velocities have a maximum of $\sim 10^{3} \mathrm{~km} \mathrm{~s}^{-1}$ in the plane $\theta=0$, and decrease to become zero at the poles, whereas rotational velocities can have a maximum, in the plane of rotation, from a few $\times 10^{3} \mathrm{~km}$ $\mathrm{s}^{-1}(\epsilon \sim 0.1)$ up to $\sim 10^{4} \mathrm{~km} \mathrm{~s}^{-1}(\epsilon \sim 1)$.

When the inclination angle is very small, the rotational component has no effect, and the only contribution from bulk motion comes from the component $v_{r} \cos \theta$ (see Equ. (10)), which gives an average contribution roughly comparable to the thermal width. However, for non-negligible values of $i$, it is the component $v_{\phi} \sin i$ that dominates the broadening of the lines (while the outer part of the wings is still further broadened by a factor roughly proportional to the thermal width $\Delta$ )

Note that, if no independent information on the inclination angle is available, the amount of rotation of the flow and the inclination angle itself cannot be constrained separately. This degeneracy can be broken in the case of M87, where observations indicate an inclination angle of $i \sim 42^{0}$ (Ford et al. 1994). Figure 3 shows the profiles of some of the strongest lines predicted for this source. Lines from elements in a high ionization state have generally broader wings, as expected, because higher ionization states are more populated at higher temperatures. The broadest line in the figure is the $\lambda 1.78$ line of Fe XXVI. Figure 4 shows, for M87, the effects of an increased rotation on the profile of the line $\lambda 1.78$. In cases such as M87, where the inclination angle is constrained by independent observations, line profiles can thus provide powerful tests

\footnotetext{
${ }^{5}$ This can be understood by considering that a line profile is obtained by the convolution of many gaussians, each centered around a value $v_{\text {los }}(r, \theta, \phi)$, and with a width $\Delta$.
} 
of the amount of rotation in the flow, and, in turn, help us learn about some of its underlying microphysics (see $\S 2.1$ ).

As already mentioned, a fit to the $X$-ray spectrum of M87 with an ADAF requires the presence of a strong wind (Di Matteo et al. 1999), which implies that some mass is lost to an outflow. Therefore we tried to estimate the effect that such an outflow might have on the line profiles. In Figure 5 we compare the profile of the line $\lambda 1.78$ with no outflow (solid line) with the profile of the same line when there is an outflow (dotted and dashed lines). The model we used for the latter is described in Appendix B. The two cases shown correspond to different parameterizations of the density. The dotted line is computed with $a=0$ in Equation (B5). This implies a constant density (with respect to $\theta$ ) and a radial component of $v_{r}$ which is comparable for the infalling mass (largest in the plane $\theta=\pi / 2$ ) and the outflowing mass (largest at the poles $\theta=0, \pi)$. The dashed line is computed with the choice $a=0.5$, correponding to lower density at the poles but higher velocity for the outflow.

We find that the presence of an outflow does not affect line profiles significantly. This can be easily understood within the framework of our model. We assumed that the outflow mainly affects $v_{r}$ and $v_{\theta}$, but not $v_{\phi}$, and that the $r$ dependence is the same as in the standard model. This resulted [cfr. Eq.(B4)] in a $v_{\theta}$ rather smaller than $v_{r}$ and $v_{\phi}$ for typical ADAF and wind parameters. The radial component of the velocity, on the other hand, can be, contrary to the standard model of pure infall, both positive and negative. However, the situation where the outflowing mass has a velocity comparable to the infalling one is very similar to the case of pure inflow. In fact, from the point of view of a distant observer, since the gas is optically thin, inflow and outflow have identical signatures if the velocities are identical. In the case in which the outflow has a higher velocity than the inflow, we do expect a difference, but the outflow has a lower density, and therefore its contribution to the total emission is not very significant .

Needless to say, the validity of these results is limited to the particular outflow model described in Appendix B. If, for example, the assumption that the outflow does not affect the rotation of the gas does not hold true, then the outflow will affect the line profiles, as Figure 4 shows.

Finally, note that optical depth effects, which could slightly modify the shape of line profiles, are not significant here. The optical depth to a line is given by $\tau \sim 0.0106 f N_{\text {ion }} / \Delta \nu$, where $f$ is the line oscillator strength and $\Delta \nu=\Delta v / \lambda$. We computed $N_{\text {ion }}$ numerically and found, for the two strongest lines that we consider, $\tau \sim 0.3$ for the OVIII $\lambda 18.21$ line, and $\tau \sim 0.02$ for the Fe XXVI $\lambda 1.87$ line, in the case of M87. For SgrA*, we found $\tau \sim 0.01$ and $\tau \sim 10^{-3}$ for the same lines, respectively.

\footnotetext{
${ }^{6}$ This feature of our model — that is the higher the velocity in the outflow the lower its density — has been imposed to mimic the results of the numerical simulations.
} 


\subsection{Other diagnostics}

Figure 6 shows the predicted $X$-ray continuum luminosity of $\mathrm{SgrA}^{*}$ for different values of the wind parameter $p$. Note that the observed flux from the region of the spectrum below $2 \mathrm{keV}$ would be highly absorbed (due to the high column density to this source), but we show the luminosity in a wider range of energy for clarity. The figure shows that the higher the value of $p$, the less emission there is at higher energies and the more at lower ones, thus making the slope steeper. This effect was pointed out by Quataert \& Narayan (1999). The difference in emission is due to the fact that the density is $\propto r^{-3 / 2+p}$, implying that, for higher $p$, there is more gas at larger radii, where the temperature is lower.

As already mentioned in the introduction, ratios between lines from low and high ionization states are very sensitive to the distribution of density with temperature in the emitting plasma. In ADAF models, this is essentially determined by the parameters $p$ and $r_{\text {tr }}$ (see Eqs. (6) and (A7)). Therefore, relative line intensities can be used as diagnostics of these two parameters. In the case of the source Sgr $\mathrm{A}^{*}$, there is no evidence of a transition to an outer disk, hence line ratios provide a useful, direct probe of the wind parameter $p$. Having an independent way (i.e. unrelated to spectral fits of the emission from the innermost part of the flow) to constrain the wind parameter in ADAF models is an important issue, as both models with and without winds fit the spectral data for Sgr A*, but require different assumptions for microscopic parameters of the flow (Quataert \& Narayan 1999).

Figure 7 shows ratios between some strong lines of iron as a function of the parameter $p$. As $p$ increases, the flow becomes overall colder (as more mass is taken away from the inner, hotter regions) and the relative concentrations of the ions of iron consequently vary, with lower ionization states becoming more populated. Ratios between two lines from different ions mostly reflect variations in their relative concentrations. These ratios have the strongest dependence on the distribution of mass with temperature (and therefore on $p$ ), as it can be seen from the dashed line (showing [Fe XXV $\lambda 1.855] /[F e$ XXVI $\lambda 1.780]$ ) and the long - dashed line (showing $[\mathrm{Fe} X X V I \lambda 1.780] /[\mathrm{Fe} X X V \lambda 1.850])$ of Figure 7. On the other hand, the ratio [Fe XXV $\lambda 1.590] /[\mathrm{Fe}$ $\mathrm{XXV} \lambda 1.850$ ] (dotted line) is sensitive to the electron temperature, due to the difference in excitation thresholds of the two lines. The fact that it is pretty flat shows that the bulk of the ions is always produced around the same temperature, and there is not a significant departure from ionization equilibrium.

Note that the ratio $[\mathrm{FeXXV} \lambda 1.867] /[\mathrm{FeXXV} \lambda 1.850]$ is very sensitive to the importance of recombination, and, in turn, to the presence of a significant photoionizing flux. Recombination to the excited levels results in a cascade which passes through the $\lambda 1.867$ transition. As a result, the intensity of such a line would be strongly enhanced with respect to its value due to only collisional excitation. When photoionization is important, the ratio [FeXXV $\lambda 1.867] /[\mathrm{FeXXV} \lambda 1.850]$ is

\footnotetext{
${ }^{7}$ Both ratios are rather insensitive to the importance of recombination.
} 
expected to be $\gtrsim 1$. Other elements have lines whose ratio would show the same behaviour, such as the ratio $[\lambda 6.740] /[\lambda 6.648]$ of the ion Si XIII. Photoionization in ADAF models was shown to be unimportant by Narayan \& Raymond, but it would be significant in other cases, such as the Compton-dominated corona models 8 . Therefore, the values of such ratios can be useful diagnostics to discriminate ADAFs from these types of accretion models?.

In the case of M87, applications of ADAF models give a much better fit to the data for high values of the wind parameter $\left(p \sim 0.4-0.5\right.$; Di Matteo et al. 1999). The transition radius $r_{\text {tr }}$ between the inner ADAF and an outer disk + corona is, however, quite uncertain. It may be possible to use relative line intensities to set constraints on $r_{\mathrm{tr}}$. Given the dependence of $\dot{M}$ on $r$ of Equation (6), it can be seen that the smaller $r_{\mathrm{tr}}$, the less gas there is at large radii, therefore resulting in an overall hotter flow. Figure 8 shows how temperature-dependent line ratios can then be used to probe the size of the ADAF region and its transition to a disk + corona. The solid line shows the ratio [Fe XXVI $\lambda 1.780] /[\operatorname{Si}$ XIV $\lambda 6.180]$; this is quite sensitive to the relative emission from different regions of the flow: Fe XXVI is produced in the very inner part whereas Si XIV is mostly abundant in the middle and outer regions. A low value of $r_{\mathrm{tr}}$, with a consequent high density in the inner part, produces an increase in the abundance of Fe XXVI compared to $\mathrm{Si}$ XIV. The ion O VIII, on the other hand, is produced at lower temperatures compared to $\mathrm{Si}$ $\mathrm{XIV}$, and this is shown in the decrease of the ratio [Si XIV $\lambda 6.180] /[\mathrm{O}$ VIII $\lambda 18.97]]$ (dotted line) with increasing $r_{\mathrm{tr}}$. The other two ratios in Figure 8, that is [Si XIII $\left.\lambda 6.648\right] /[\mathrm{Si}$ XIV $\lambda 6.180$ ] (dashed line) and [Fe XXV $\lambda 1.850] /$ [Fe XXVI $\lambda 1.780]$ (dotted - dashed line), are mostly sensitive to the relative ionic concentrations. As the flow is not too far from equilibrium, these ratios are sensitive to the distribution of mass with radius. If $r_{\mathrm{tr}}$ is small, more mass is concentrated in the inner and hotter regions, thus making the higher ionization states of the elements more populated. This point is further illustrated by Figure 9, which shows the emissivity of some of the strongest lines as a function of radius for two different values of $r_{\mathrm{tr}}$, i.e. $r_{\mathrm{tr}}=10^{4}$ and $r_{\mathrm{tr}}=10^{5}$. While the temperature profile is the same in both cases, the density profile is different, with more mass in the outer region for the case of $r_{\mathrm{tr}}=10^{5}$ (see Equ. (6)) than for the case of $r_{\mathrm{tr}}=10^{4}$. Lines produced in the outer region are therefore enhanced compared to lines produced in the inner part of the flow, if larger values of $r_{\mathrm{tr}}$ are assumed.

\subsection{Observational prospects}

Table 1 shows the luminosities of some of the most important lines for both Sgr $\mathrm{A}^{*}$ and M87. For each source, we considered one model without wind $(p=0)$, and one with a moderate

\footnotetext{
${ }^{8}$ Typical spectra of photoionization-dominated emission are those from $X$-ray low-mass binaries, and they show very different signatures from those discussed here (see e.g. the case of Cyg X3 discussed by Liedhal \& Paerels 1996).

${ }^{9}$ In photoionization-dominated accretion models, however, the overall intensity of all lines would be lower, as pointed out by Narayan \& Raymond (1999).
} 
wind $(p=0.4)$. Equivalent widths (EWs) for the same lines have been computed by Narayan \& Raymond. For M87 and a wind model, the strongest lines at both low and high energies have typical EWs $\gtrsim$ a few tens of $\AA$. This is the case also for the strongest iron lines in the models of Sgr $A^{*}$. In the case of this source, these would be the only observable lines, due to the high column density $\left(N_{\mathrm{H}} \sim 6 \times 10^{22} \mathrm{~cm}^{-2}\right)$, which causes a strong absorption of the emission below about $2 \mathrm{keV}$. This is not a problem for M87, where the column density is much lower $\left[N_{\mathrm{H}}=(2.1 \pm 0.3) \times 10^{20} \mathrm{~cm}^{-2}\right.$, Sankrit, Sembach, \& Canizares 1999]. Given the distances to these sources (8.5 kpc for Sgr $\mathrm{A}^{*}$ and $16 \mathrm{Mpc}$ for M87), the brightest lines are already within the detection capability of the ACIS detector on the Chandra X-ray Observatory (CXO). With an exposure of $10^{5} \mathrm{sec}$, the flux threshold is $\sim 2 \times 10^{-14} \mathrm{erg} \mathrm{cm}^{-2} \mathrm{sec}^{-1}$ around $6 \mathrm{keV}$ (where most of the iron lines are) and $\sim 6 \times 10^{-16} \mathrm{erg} \mathrm{cm}^{-2} \mathrm{sec}^{-1}$ at an energy $\sim 0.6 \mathrm{keV}$ (where the strong $\mathrm{O}$ VIII line is). The higher-energy threshold corresponds to a luminosity of $\sim 6 \times 10^{38} \mathrm{ergs} \mathrm{s}^{-1}$ for M87 and $\sim 2 \times 10^{32} \operatorname{ergs~s}^{-1}$ for Sgr A* (without correction for absorption), while the limiting flux at $0.6 \mathrm{keV}$ corresponds to $L \sim 2 \times 10^{37} \mathrm{ergs} \mathrm{s}^{-1}$ for M87. Lines in this range of the spectrum are highly absorbed for Sgr $\mathrm{A}^{*}$.

For measurements of line profiles, the spectral resolution of HETG on CXO is $\Delta E / E \sim 5 \times 10^{-3}$ around $6 \mathrm{kev}$, while the LETG spectral resolution is $\Delta E / E \sim 2.5 \times 10^{-3}$ at about $0.6 \mathrm{keV}$, which corresponds to $\Delta v \sim 700 \mathrm{~km} \mathrm{~s}^{-1}$. Given the inclination angle inferred for M87, this resolution should be sufficient to constrain the rotational velocity of the gas (see Figure 4), if a sufficient number of counts is available for the line under consideration. We used the Chandra Proposal Planning Toolkit to process the flux from the OVIII $\lambda 18.97$ line predicted for M87 (while taking also absorption into account) with the ACIS-S HETG/MEG1 detector, and found $\sim 4$ counts/ksec. This yields about 400 counts after a typical integration time of $100 \mathrm{ksec}$. The statistics should therefore be large enough to allow an estimate of the line widths, though not a detailed profile measurement. Excellent spectra for the low energy lines will also be provided by $X M M$.

The above estimates are based on models which have been fitted to the present best estimates of the $X$-ray fluxes of Sgr $\mathrm{A}^{*}$ and M87. Observations with $C X O$ at high angular resolution will provide more accurate $X$-ray fluxes, which may lead to modest revision in the estimated line intensities.

\section{Conclusions}

We have shown that high-resolution observations of line profiles have the potential to probe the dynamics of thermal gas in accretion flows around black holes. Line profiles are, essentially, indicators of the temperature of the emitting plasma and of its velocity, but they also depend on the angle between the line of sight to the observer and the axis of rotation of the flow. In the standard ADAF model, velocities are independent of the mass of the central object and, consequently, line profiles are also independent of it. For non-negligible values of the inclination angle, we find that line broadening is dominated by the bulk rotational motion of the gas. We 
have demonstrated that in cases, such as M87, where there is independent evidence of the value of the inclination angle, the amount of rotation of the flow can be well constrained.

We have shown that further knowledge of properties of the flow can be obtained through measurements of line ratios and the slope of the continuum, which are sensitive to the distribution of mass with temperature in the flow. In standard ADAF models, this is primarily determined by the amount of mass lost to a wind, and by the size of the hot region. We have presented our results for ADAFs applied to the sources Sgr $\mathrm{A}^{*}$ and M87. In the case of Sgr $\mathrm{A}^{*}$, where there is no evidence of an outer disk, we have illustrated how relative line intensities can provide strong indications of the mass lost to a wind during accretion. This is particularly useful for this source, where the wind parameter is still uncertain and cannot be constrained by spectral fits. In the case of M87, where models with winds provide a better fit to the data, we have showed that line ratios can be powerful probes of the transition radius between the ADAF and an outer disk + corona. Finally, we have presented line ratio diagnostics that can help distinguish ADAFs from photoionization-dominated accretion models. We find that the strongest lines are within the detection capability of current $X$-ray instruments.

\section{REFERENCES}

Abramowicz, M., Chen., Kato, S., Lasota, J.-P., \& Regev, O. 1995, ApJ, 438, L37

Arnaud, M. \& Raymond, J. 1992, ApJ, 398, 394

Bely-Daubau, F., Faucher, P., Dubau, J., \& Gabriel, A. H. 1982, MNRAS, 198, 239

Blandford, R. D., Begelman, M. C. 1999, MNRAS, 303, L1

Coker, R. F., \& Melia, F. 1997, ApJ, 488, L149

Di Matteo, T., Fabian, A. C., Rees, M. J., Carilli, C. L., Ivison, R. J. 1999, MNRAS, 305, 492

Dubau, J., Loulergue, M., Gabriel, A. H., Steenman-Clark, L., \& Volonte, S. 1981, MNRAS, 195, 705

Esin, A. A., McClintock, J. E., \& Narayan, R. 1997, ApJ, 489, 867

Ford, H. C. et al., 1994, ApJ, 435, L27

Igumenshchev, I. V. astro-ph/9902259

Igumenshchev, I. V. \& Abramowicz, M. A. 1999, MNRAS, 303, 309

Igumenshchev, I. V. \& Abramowicz, M. A. , 2000, astro-ph/0003397

Liedahl, D. A. \& Paerels, F. 1996, ApJ, 468, 33L

Kato, S., Fukue, J., \& Mineshige, S. 1998, Black Hole Accretion Disks (Kyoto: Kyoto Univ. Press)

Liang, E. P. 1998, Phys. Rep., 302, 67

Mewe, R., Schrjiver, J., \& Sylwester, J. 1980, A\&AS, 40, 323 
Mushotzky, R. F., Done, C., \& Pounds, K. A. 1993, ARA\& A, 31, 717

Narayan, R. \& Yi, I. 1994, ApJ, 428, L13

Narayan, R. \& Yi, I. 1995a, ApJ, 444, 231

Narayan, R. \& Yi, I. 1995, ApJ, 452, 710

Narayan, R. \& Raymond, J. 1999, ApJ, 515, L69

Narayan, R., Igumenshchev, I. V. \& Abramowicz, M. A., 2000, apJ in press, astro-ph/9912449

Narayan, R., Mahadevan, R., \& Quataert, E. 1998, in Theory of Black Hole Accretion Disks, eds. M. A. Abramowicz, G. Bjornsson, \& J. E. Pringle (Cambridge: Cambridge Univ. Press)

Pradhan, A. K. 1985, ApJS, 59, 183

Pradhan, A. K., Norcross, D. W., \& Hummer, D. G. 1981, ApJ, 246, 1031

Quataert, E. \& Narayan, R. 1999, ApJ, 520, 298

Quataert, E. \& Narayan, R. 2000, ApJ in press, astro-ph/9908199

Quataert, E., Narayan, R. \& Reid, M. J. 1999, ApJ, 517, L101

Quataert, E., \& Gruzinov, A. 2000, ApJ in press, astro-ph/9912440

Raymond, J. \& Smith, B. W. 1977, ApJS, 35, 419

Reynolds, C. S., Di Matteo, T., Fabian, A. C., Hwang, U., \& Canizares, C. R. 1996, MNRAS, 283, L11

Sankrit, R., Sembach, K. R., \& Canizares, C. R. 1999, ApJ in press 


\section{APPENDIX}

\section{A. $\theta$ dependence of the density profile}

Under the assumption that $v_{\theta}=0$ and the viscosity terms can be neglected, the equation of hydrostatic equilibrium in the $\theta$ direction reduces to

$$
\frac{1}{r} \frac{d P}{d \theta}=\rho v_{\phi}^{2} \frac{\cot \theta}{r} .
$$

Recalling that $p=c_{s}^{2} \rho$, with $\rho(r, \theta)=\rho(\theta) r^{-3 / 2}$, Equation (A1) gives

$$
\frac{1}{\rho(\theta)} \frac{d}{d \theta}\left[\rho(\theta) c_{s}^{2}(\theta)\right]=v_{\phi}^{2} \cot \theta
$$

Using Equations (3) and (4), this becomes

$$
\frac{d \rho}{\rho}=\left(c_{3}+2 c_{2}\right) \frac{\sin \theta \cos \theta}{c_{1}-c_{2} \sin ^{2} \theta} d \theta,
$$

having defined $c_{3} \equiv 2 \epsilon /(5+2 \epsilon)$. Equation $(\mathrm{A} 3)$ can be integrated to yield

$$
\rho(\theta)=\rho_{0}\left(\frac{1}{c_{1}-c_{2} \sin ^{2} \theta}\right)^{\frac{c_{4}}{2 c_{2}}}
$$

where $c_{4} \equiv c_{3}+2 c_{2}=3.6 \epsilon /(5+2 \epsilon)$. Finally, noticing that $c_{4} / 2 c_{2}=2.25$ and using Equation (四), we get

$$
\rho(\theta)=\rho_{0}\left(c_{s}^{2}\right)^{-2.25}
$$

The constant of integration $\rho_{0}(r)$ depends on the mass accretion rate through the equation (e.g. Narayan \& Yi 1995a)

$$
\dot{M}(r)=-\int 2 \pi r^{2} \sin \theta \rho(r, \theta) v_{r}(r, \theta) d \theta
$$

which yields

$$
\rho_{0}(r)=-\frac{\dot{M}(r)}{2 \pi f \sqrt{G M}},
$$

where $f \equiv[-3 \alpha /(5+2 \epsilon)] \int_{0}^{\pi} d \theta\left(c_{s}^{2}\right)^{-2.25} \sin ^{3} \theta$.

\section{B. Simplified model of an ADAF with an outflow}

We consider a self-similar flow in which the $r$ dependences of density, sound speed and components of the velocity (including $v_{\theta}$ ) are the same as in the pure ADAF with no outflow. All variables are assumed to be independent of $\phi$ as before, and the amount of rotation (expressed through $v_{\phi}$ ) is also left unaltered. What we need to find is therefore the $\theta$ dependence of 
$v_{r}, v_{\theta}, \rho, c_{s}$. We parameterize two of them, and find the other two from the continuity equation and the momentum equation in the $\theta$ direction. We look for a solution in which there is pure inflow in the equatorial plane $\theta=\pi / 2$, and pure outflow at the poles. Therefore we require $v_{\theta}(0)=v_{\theta}(\pi)=0$. We also want a $v_{\theta}$ which changes sign in going from the upper to the lower part of the plane of rotation. The numerical simulations of Igumenshchev and Abramovicz (1999) also suggest that higher velocities are accompanied by lower densities and vice versa. The conditions required above are satisfied by the simple function

$$
\rho(r, \theta) v_{\theta}(r, \theta)=\rho_{0} v_{\theta}^{0} r^{p-2} \sin (2 \theta) .
$$

For the $r$ component of the momentum we look for something of the type $\rho(r, \theta) v_{r}(r, \theta)=$ $\rho_{0} v_{r}^{0} r^{p-2} f(\theta)$; the continuity equation

$$
\frac{1}{r^{2}} \frac{\partial}{\partial r}\left(r^{2} \rho v_{r}\right)+\frac{1}{r \sin \theta} \frac{\partial}{\partial \theta}\left(\sin \theta \rho v_{\theta}\right)=0
$$

constrains the functional form of $f(\theta)$ together with the relation between $v_{r}^{0}$ and $v_{\theta}^{0}$. That is,

$$
\rho(r, \theta) v_{r}(r, \theta)=\rho_{0} v_{r}^{0} r^{p-2}\left[\cos (2 \theta)+\cos ^{2} \theta\right]
$$

and

$$
v_{\theta}^{0}=\frac{v_{r}^{0} p}{2}
$$

At any given $r$, the value of $v_{r}^{0}$ represents the (pure infall) velocity in the $\theta=\pi / 2$ plane, and thus we choose it as in Equation (1). The value of $v_{\theta}^{0}$ is then determined by Equation (B4). The constant $\rho_{0}$ is determined so that the total mass of the flow is the same as in the case with no outflow.

Next we parameterize the density. Igumenshchev \& Abramowicz (1999) have presented numerical simulations with an outflow, which show that the density is maximum in the plane of rotation and decreases towards the poles. This can be represented by the function

$$
\rho(\theta)=\rho_{0}\left(1-a \cos ^{2} \theta\right),
$$

where $\rho_{0}$ is the value of the density in the plane and $a \equiv\left(1-\rho_{\min } / \rho_{0}\right)$ expresses the degree of variation of the density with angle. Finally, the sound speed is determined from the momentum equation in the $\theta$ direction. Neglecting the viscosity terms, this gives

$$
\rho\left[v_{r} \frac{\partial v_{\theta}}{\partial r}+\frac{v_{\theta}}{r} \frac{\partial v_{\theta}}{\partial \theta}+\frac{v_{r} v_{\theta}}{r}-\frac{v_{\phi}^{2} \cot \theta}{r}\right]=-\frac{1}{r} \frac{\partial P}{\partial \theta} .
$$

If the density does not decrease too steeply towards the poles (in the standard ADAF with no outflow this is the case for small $\epsilon$ ), then the first three terms in the left-hand side of Equation (B6) are negligible compared to the fourth term. This can be seen using the condition (B4) together with the coefficients of Equations (1) and (3), with $\epsilon=0.1, \alpha=0.1$. The $\theta$ component of 
the pressure is then given by $p(\theta)=\rho_{0} c_{3}\left[-0.5 \cos ^{2} \theta+0.25 a \cos ^{4} \theta\right]+$ const. The constant of integration is determined by imposing that the sound speed $c_{s}=\sqrt{p / \rho}$ has the same value as in Equation (4) in the plane $\theta=\pi / 2$. Once the sound speed is obtained, the temperature can then be determined as usual from Equation (7).

Finally, when $v_{\theta} \neq 0$, Equation (10) for the component of the velocity along the line of sight (needed for the computation of line profiles) is generalized to

$$
\begin{aligned}
v_{\mathrm{los}}(r, \theta, \phi) & =v_{r}(r, \theta) \sin \theta \cos \phi \sin i+v_{r}(r, \theta) \cos \theta \cos i-v_{\phi}(r, \theta) \sin i \sin \phi \\
& +v_{\theta}(r, \theta) \cos \theta \cos \phi \sin i-v_{\theta}(r, \theta) \sin \theta \cos i .
\end{aligned}
$$




\begin{tabular}{|c|c|c|c|c|}
\hline Line & SNW & SW & MNW & MW \\
\hline O VII $\lambda 21.60$ & $2.2 \times 10^{31}$ & $2.6 \times 10^{32}$ & $5.9 \times 10^{36}$ & $2.4 \times 10^{38}$ \\
O VIII $\lambda 18.87$ & $2.3 \times 10^{33}$ & $1.9 \times 10^{34}$ & $6.2 \times 10^{38}$ & $1.7 \times 10^{40}$ \\
Mg XI $\lambda 9.168$ & $1.3 \times 10^{32}$ & $1.5 \times 10^{33}$ & $3.4 \times 10^{37}$ & $1.3 \times 10^{39}$ \\
Mg XII $\lambda 8.425$ & $8.1 \times 10^{32}$ & $7.1 \times 10^{33}$ & $2.1 \times 10^{38}$ & $6.4 \times 10^{39}$ \\
Si XIII $\lambda 6.648$ & $6.7 \times 10^{32}$ & $7.2 \times 10^{33}$ & $1.7 \times 10^{38}$ & $6.6 \times 10^{39}$ \\
Si XIV $\lambda 6.180$ & $1.7 \times 10^{33}$ & $1.3 \times 10^{34}$ & $4.6 \times 10^{38}$ & $1.2 \times 10^{40}$ \\
S XV $\lambda 5.039$ & $6.1 \times 10^{32}$ & $5.7 \times 10^{33}$ & $1.6 \times 10^{38}$ & $5.1 \times 10^{39}$ \\
S XVI $\lambda 4.727$ & $1.0 \times 10^{33}$ & $6.1 \times 10^{33}$ & $2.7 \times 10^{38}$ & $5.5 \times 10^{39}$ \\
Ar XVII $\lambda 3.949$ & $4.1 \times 10^{32}$ & $3.2 \times 10^{33}$ & $1.0 \times 10^{38}$ & $2.9 \times 10^{39}$ \\
Ar XVIII $\lambda 3.731$ & $5.8 \times 10^{32}$ & $2.5 \times 10^{33}$ & $1.5 \times 10^{38}$ & $2.3 \times 10^{39}$ \\
Ca XIX $\lambda 3.173$ & $1.2 \times 10^{32}$ & $7.8 \times 10^{32}$ & $3.2 \times 10^{37}$ & $7.0 \times 10^{38}$ \\
Ca XX $\lambda 3.020$ & $1.6 \times 10^{32}$ & $5.3 \times 10^{32}$ & $4.2 \times 10^{37}$ & $4.8 \times 10^{38}$ \\
Fe XXIV $\lambda 1.861$ & $3.3 \times 10^{33}$ & $1.1 \times 10^{34}$ & $8.8 \times 10^{38}$ & $1.0 \times 10^{40}$ \\
Fe XXV $\lambda 1.850$ & $1.2 \times 10^{33}$ & $4.2 \times 10^{33}$ & $3.3 \times 10^{38}$ & $3.8 \times 10^{39}$ \\
Fe XXV $\lambda 1.867$ & $1.2 \times 10^{33}$ & $4.5 \times 10^{33}$ & $3.2 \times 10^{38}$ & $4.1 \times 10^{39}$ \\
Fe XXV $\lambda 1.859$ & $6.1 \times 10^{32}$ & $1.9 \times 10^{33}$ & $1.5 \times 10^{38}$ & $1.7 \times 10^{39}$ \\
Fe XXV $\lambda 1.590$ & $1.0 \times 10^{33}$ & $5.3 \times 10^{33}$ & $2.6 \times 10^{38}$ & $4.8 \times 10^{39}$ \\
Fe XXV $\lambda 1.855$ & $5.1 \times 10^{31}$ & $2.7 \times 10^{32}$ & $1.3 \times 10^{37}$ & $2.4 \times 10^{38}$ \\
Fe XXV $\lambda 1.780$ & $3.7 \times 10^{33}$ & $6.0 \times 10^{33}$ & $9.8 \times 10^{38}$ & $5.4 \times 10^{39}$ \\
Fe XXV $\lambda 1.500$ & $7.4 \times 10^{32}$ & $1.0 \times 10^{33}$ & $1.9 \times 10^{38}$ & $9.2 \times 10^{38}$ \\
Ni XXVII $\lambda 1.587$ & $1.8 \times 10^{32}$ & $4.9 \times 10^{32}$ & $4.7 \times 10^{37}$ & $4.4 \times 10^{38}$ \\
Ni XXVIII $\lambda 1.530$ & $1.8 \times 10^{32}$ & $2.4 \times 10^{32}$ & $4.9 \times 10^{37}$ & $2.2 \times 10^{38}$ \\
\hline
\end{tabular}

Table 1. Luminosities of some of the strongest lines for Sgr $\mathrm{A}^{*}$ without wind (SNW) and a moderate wind parameter of $p=0.4(\mathrm{SW})$, and for M87 without wind (MNW) and with $p=0.4$ (MW). 

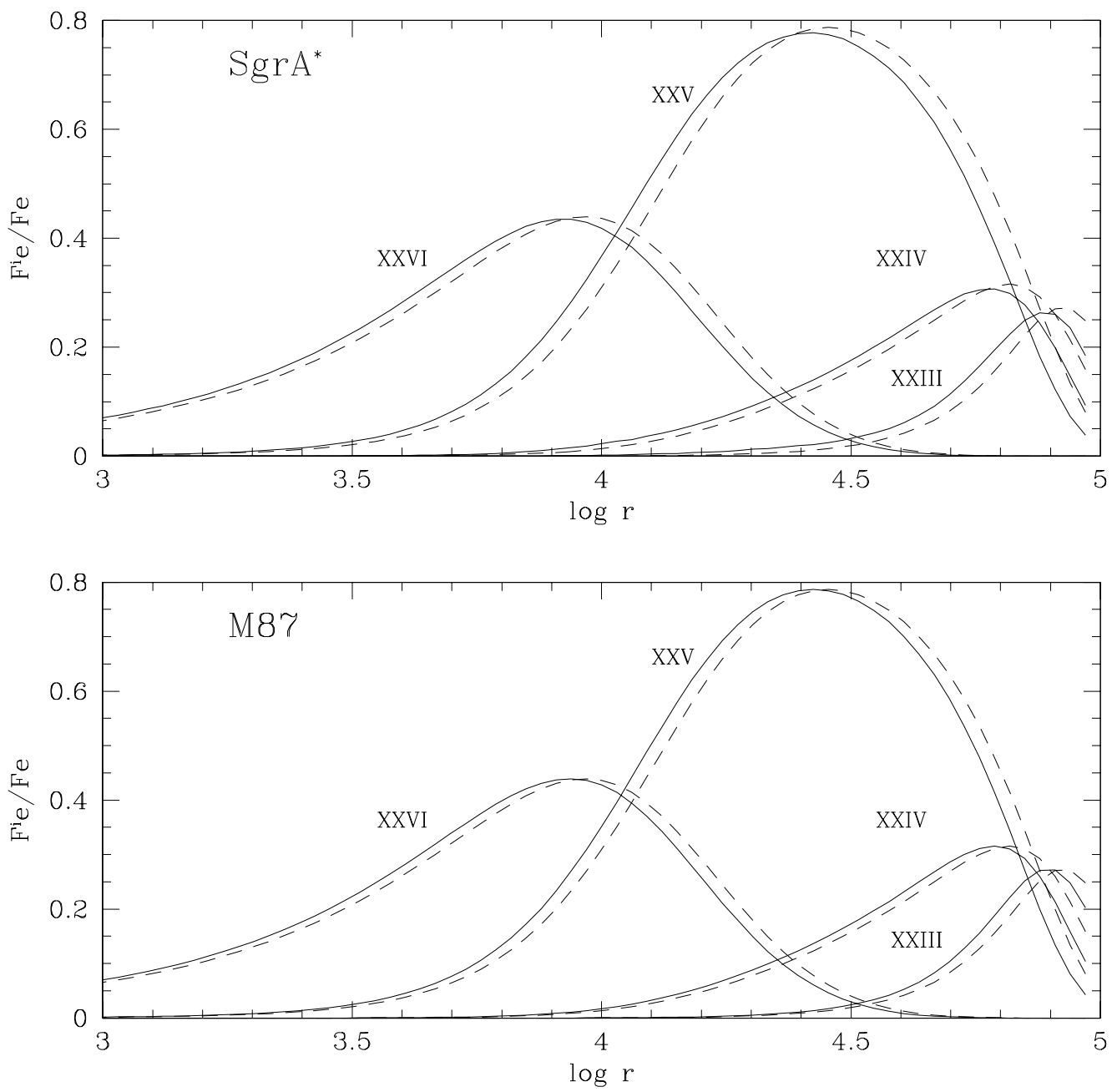

Fig. 1. - The effects of the departure from ionization equilibrium are shown for some of the important ions of iron in the two sources Sgr $A^{*}$ and M87. In both panels, the dashed lines represent the ionic concentrations at equilibrium, whereas the solid lines show the non-equilibrium concentrations. 


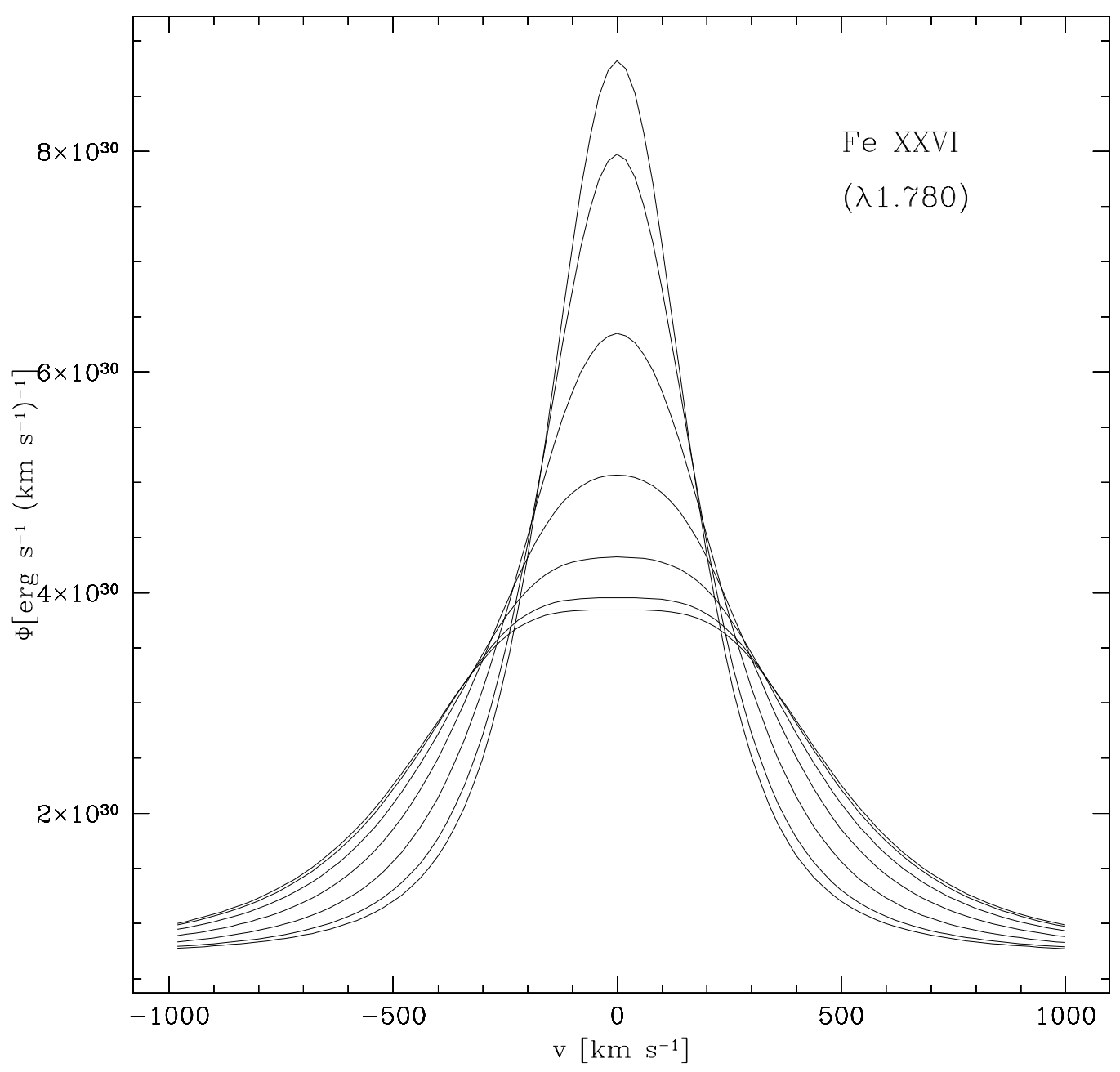

Fig. 2.- Line profiles for different choices of the inclination angle $i$ of the line of sight with respect to the axis of rotation of the flow, for the Galactic center source SgrA*. The narrowest line corresponds to $i=0^{0}$; the other lines (in order of increasing broadening) are computed with increments of $15^{0}$. The broadest line corresponds to $i=90^{\circ}$. All the profiles have been computed for $p=0$ and $\epsilon=0.1$. 


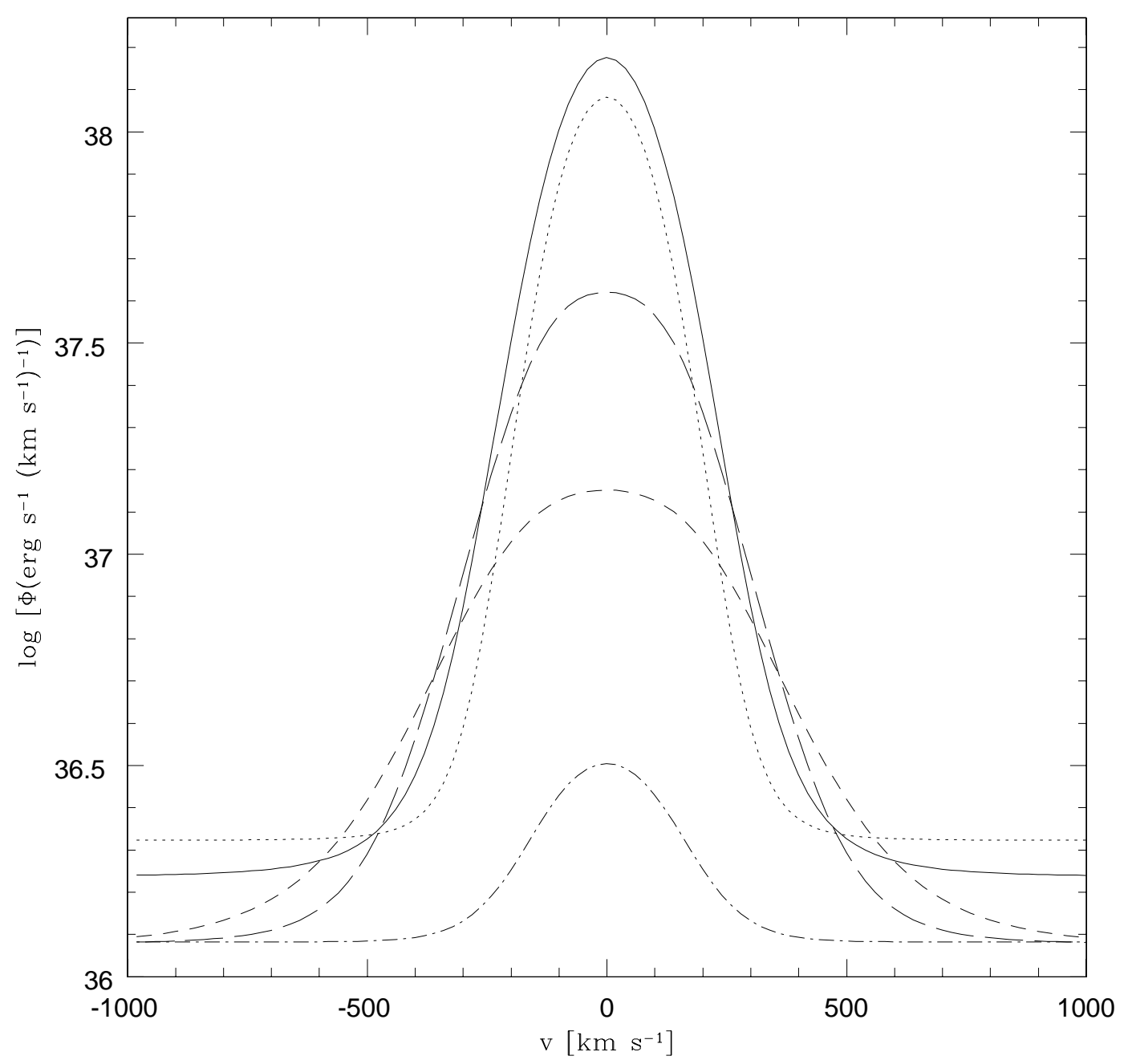

Fig. 3. - Line profiles of some of the strongest lines for the source M87: O VIII $\lambda 18.97$ (solid line), Si XIII $\lambda 6.648$ (dotted line), Fe XXIV $\lambda 1.861$ (dotted - dashed line), Fe XXV $\lambda 1.850$ (long - dashed line), Fe XXVI $\lambda 1.780$ (dashed line). The broadening of a line reflects the temperature of the region in which it is produced; the intensity is proportional to the amount of gas at that temperature. Here the parameters are $p=0.5, \epsilon=0.1, r_{\mathrm{tr}}=10^{5}$ and $i=42^{0}$. 


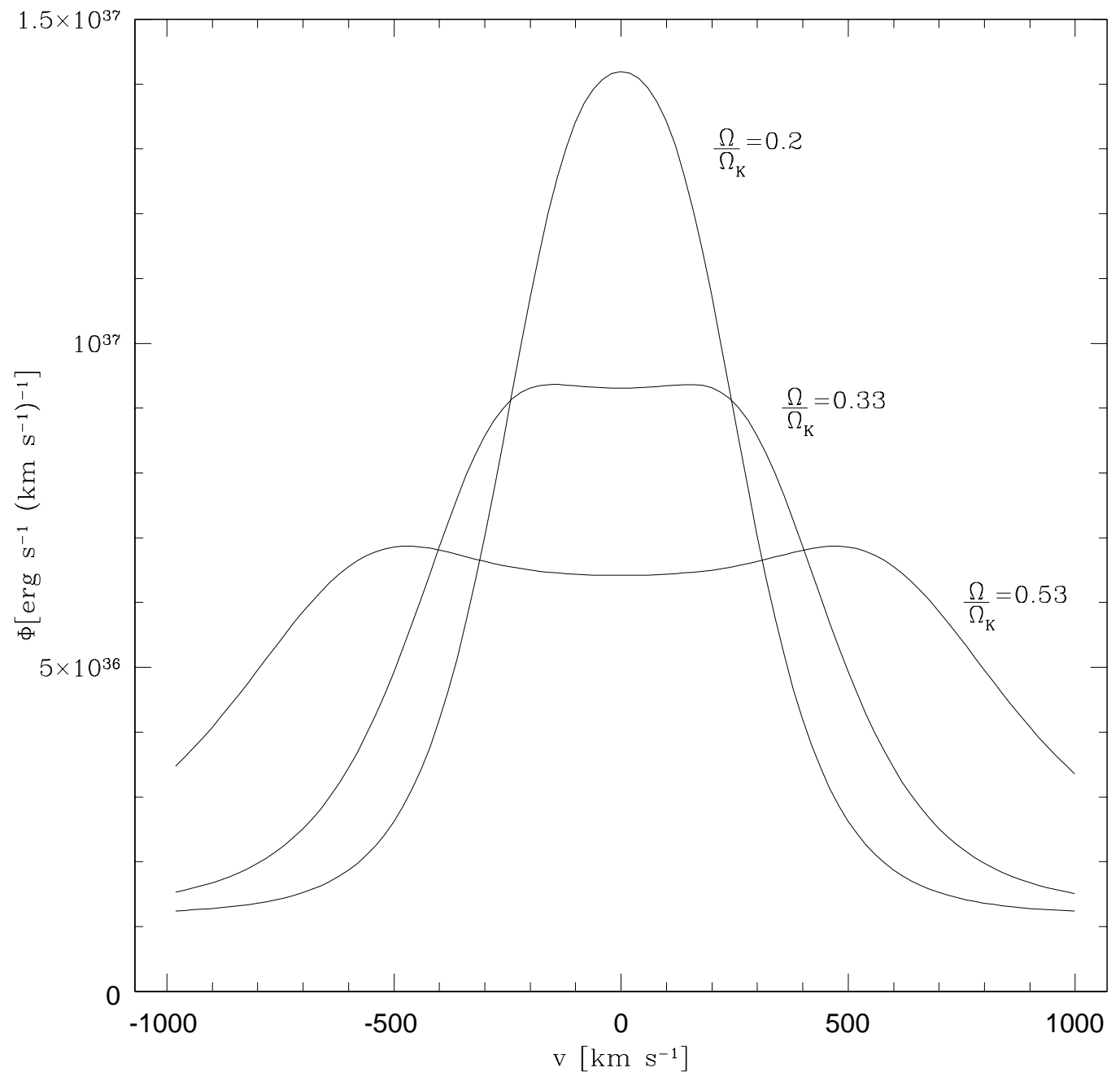

Fig. 4.- Line profile of the line Fe XXVI $\lambda 1.780$ for the source M87. Here we assume $p=0.5$, $r_{\mathrm{tr}}=10^{5}, i=42^{0}$, and show the variation of the profile with the amount of rotation of the flow. In the self-similar ADAF model that we are using, $\Omega / \Omega_{\mathrm{K}}=0.2$ corresponds to $\epsilon=0.1, \Omega / \Omega_{\mathrm{K}}=0.33$ to $\epsilon=0.3$, and $\Omega / \Omega_{\mathrm{K}}=0.53$ to $\epsilon=1$. 


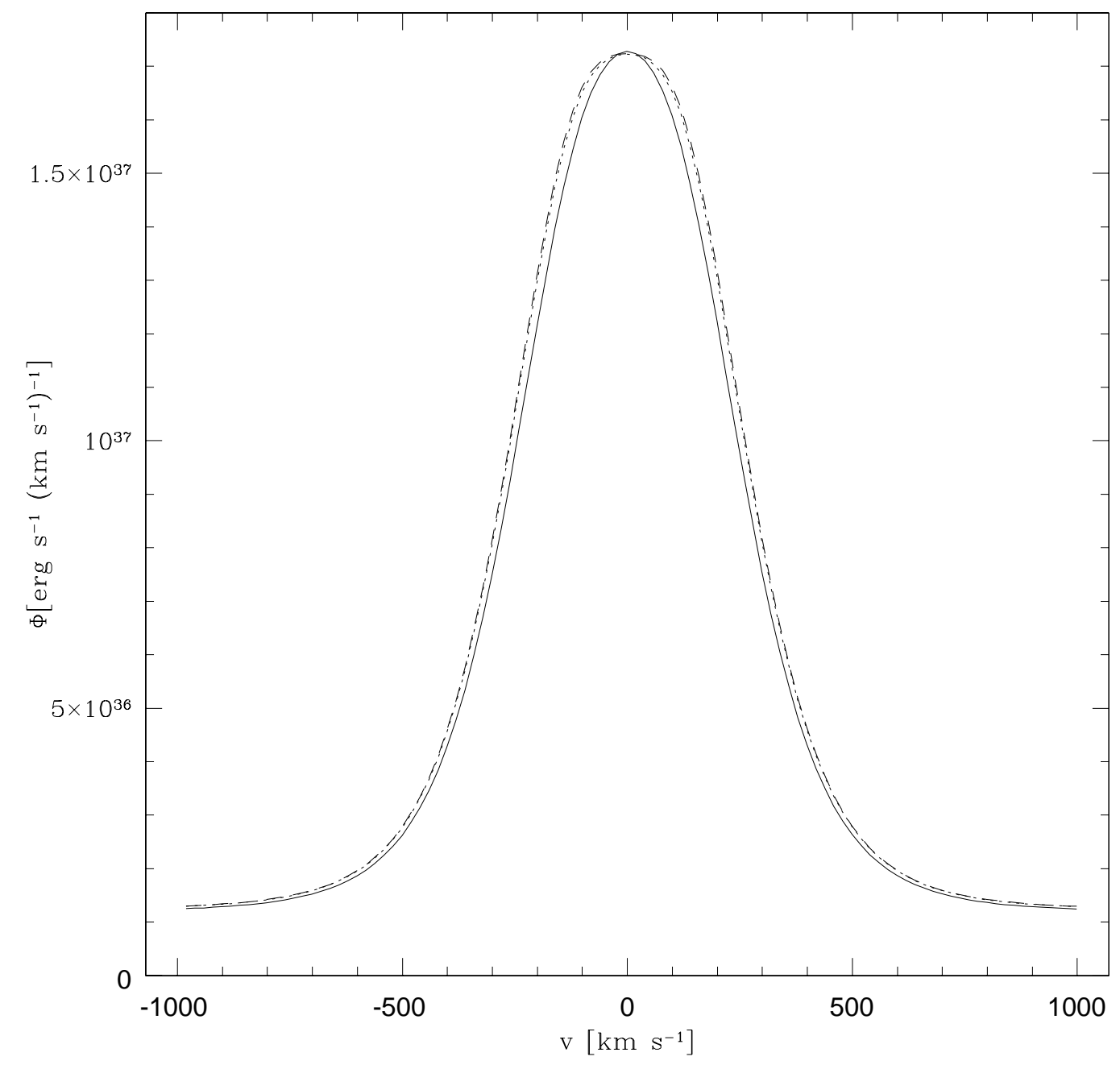

Fig. 5. - Effects of an outflow on the profile of the line Fe XXVI $\lambda 1.780$. The solid line is calculated with no outflow, while the other two lines correspond to an outflow model with $a=0$ (dotted line) and $a=0.5$ (dashed line). The source here is M87 and the model parameters are as in Figure 4 with $\epsilon=0.1$. In all cases, the concentrations are calculated at equilibrium. 


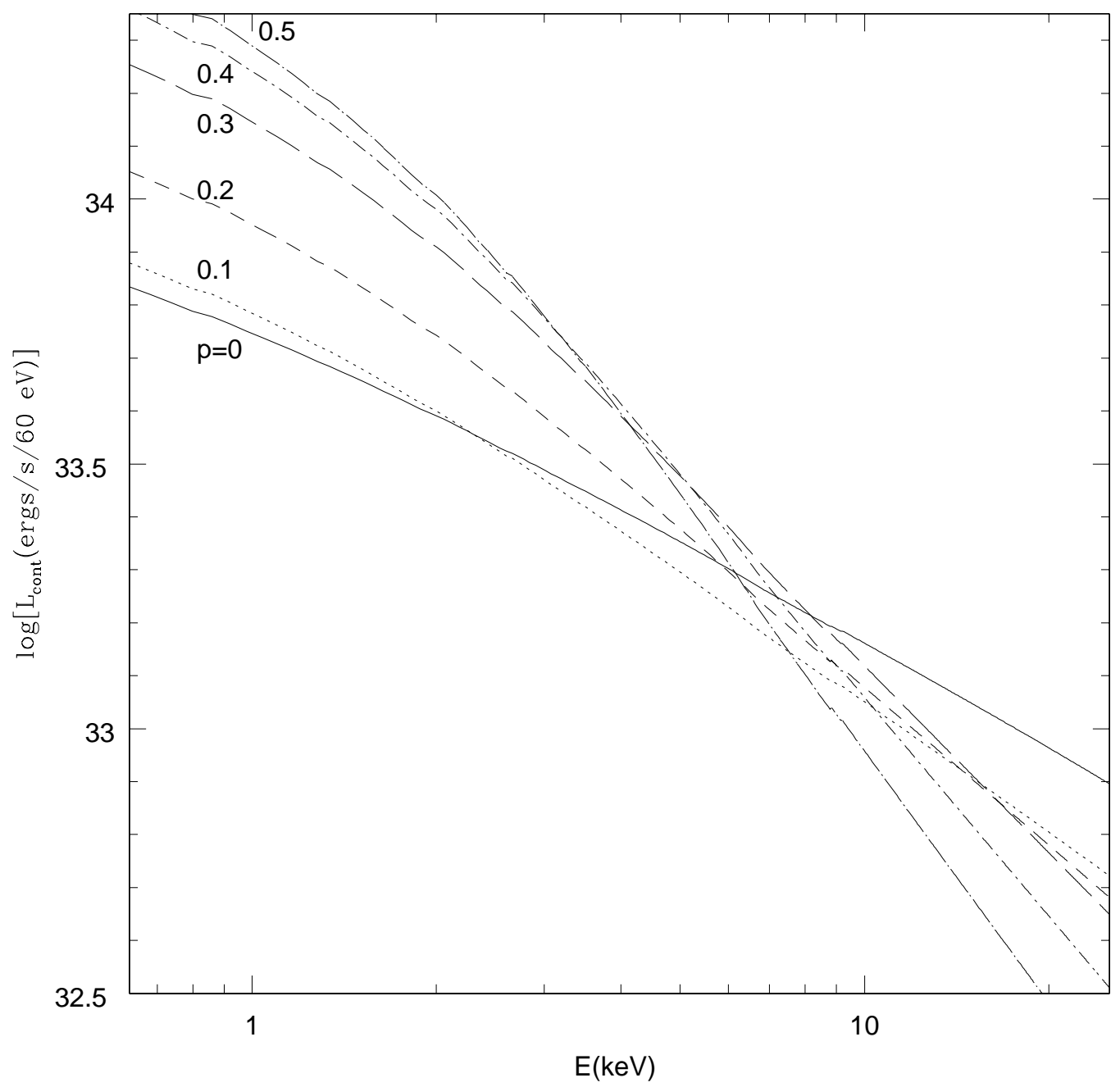

Fig. 6.- $X$-ray continuum of SgrA* for different values of the wind parameter $p$. The higher the wind, the colder the gas, and consequently the higher is the emission at low energies compared to high energies, resulting in a steeper slope. 


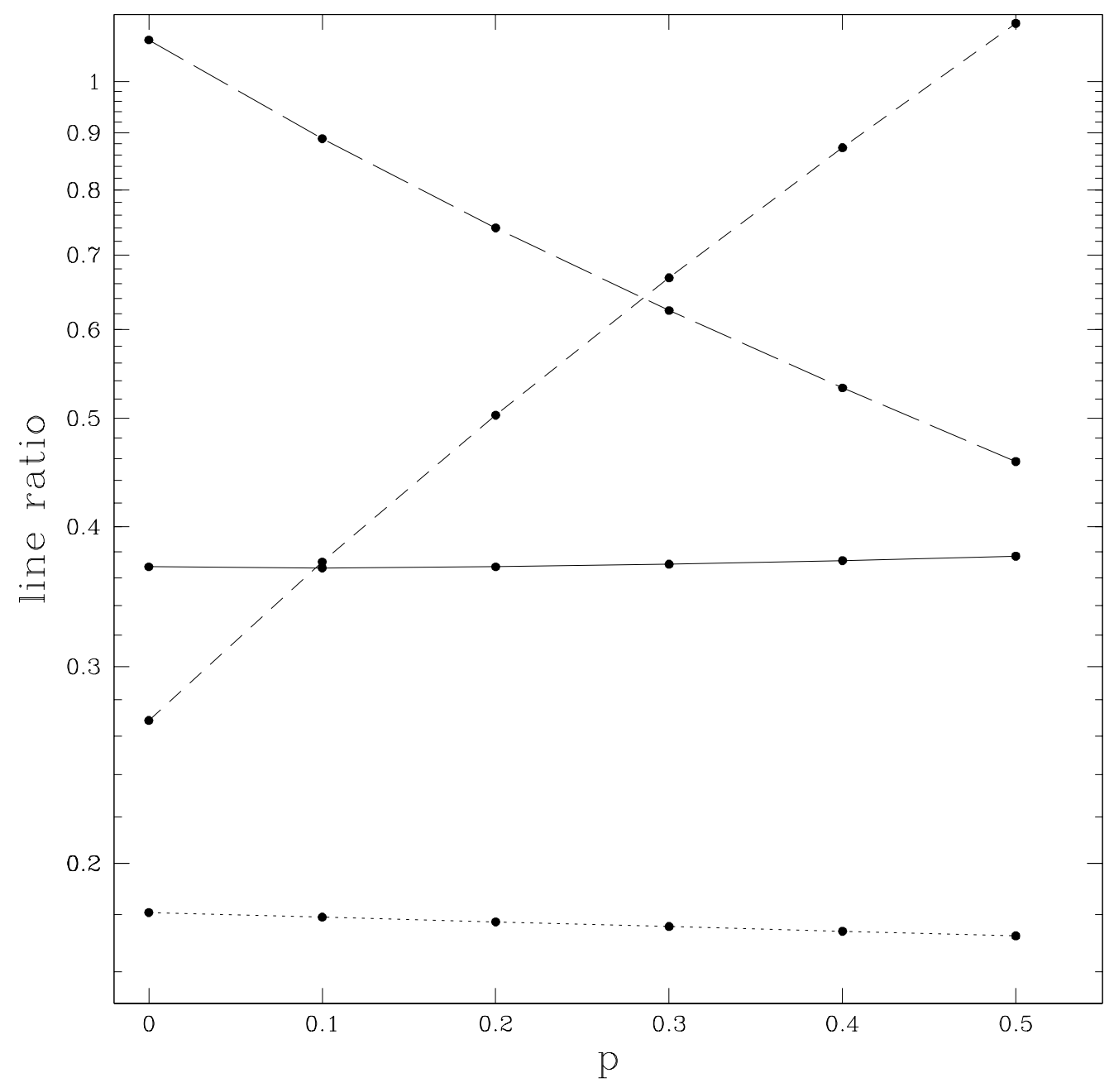

Fig. 7.- Line diagnostics of the wind parameter $p$ for SgrA*. The lines are the following: [Fe $\mathrm{XXV} \lambda 1.867] /[\mathrm{Fe} \mathrm{XXV} \lambda 1.850]$ (solid line), [Fe XXV $\lambda 1.590] /[\mathrm{Fe} \mathrm{XXV} \lambda 1.850]$ (dotted line), [Fe XXV $\lambda 1.855] /[F e X X V I \lambda 1.780]$ (dashed line), [Fe XXVI $\lambda 1.780] /[$ Fe XXV $\lambda 1.850]$ (long - dashed line). 


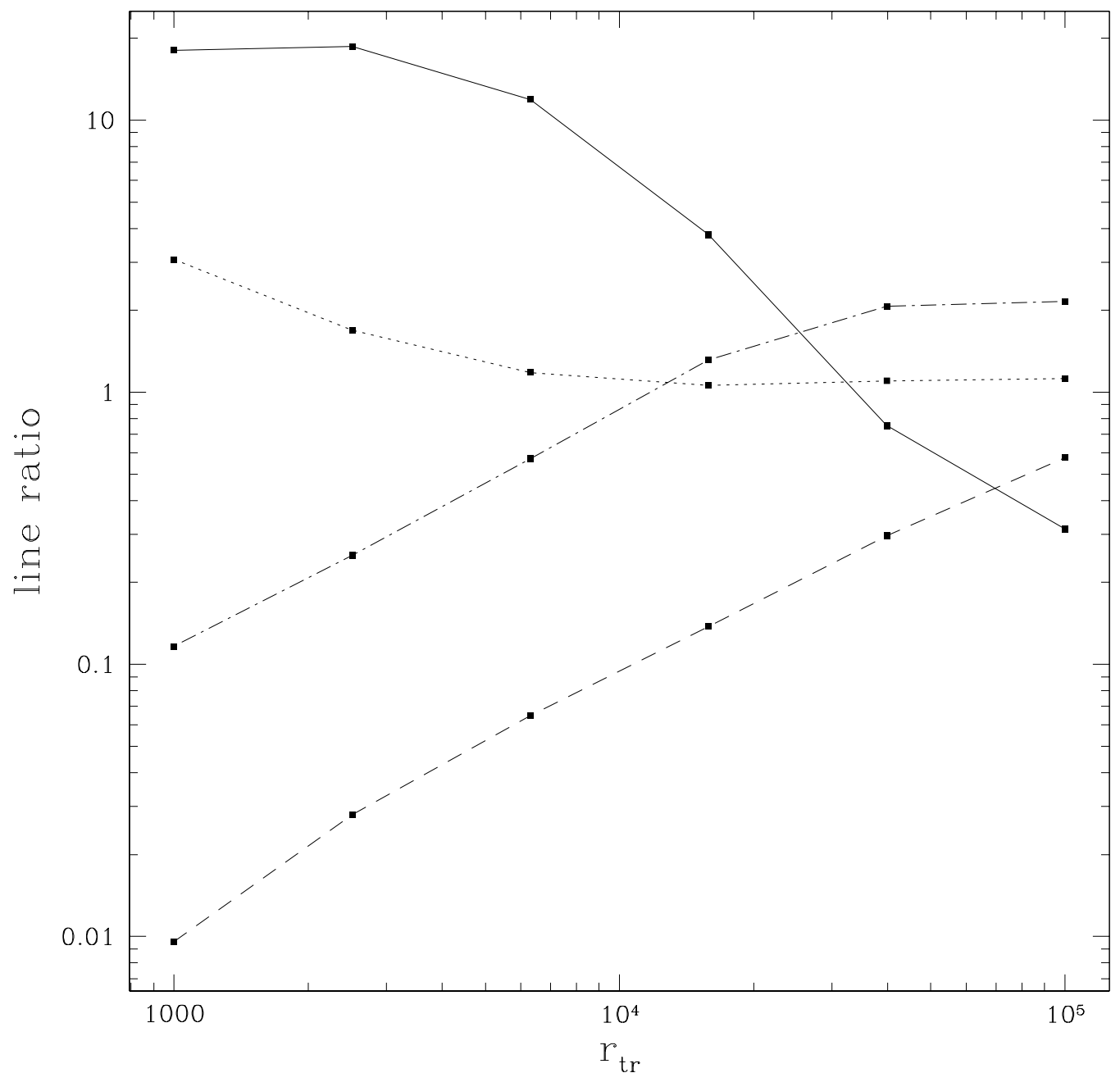

Fig. 8. - Line diagnostics of the transition radius between an ADAF and a disk + corona for M87. The lines are the following: [Fe XXVI $\lambda 1.780] /[S i$ XIV $\lambda 6.180]$ (solid line), [Si XIV $\lambda 6.180] /[\mathrm{O}$ VIII $\lambda 18.97]$ ] (dotted line), [Si XIII $\lambda 6.648] /[\mathrm{Si}$ XIV $\lambda 6.180]$ (dashed line), [Fe XXV $\lambda 1.850] /[\mathrm{Fe}$ XXVI $\lambda 1.780]$ (dotted - dashed line). Here the wind parameter is $p=0.5$. 

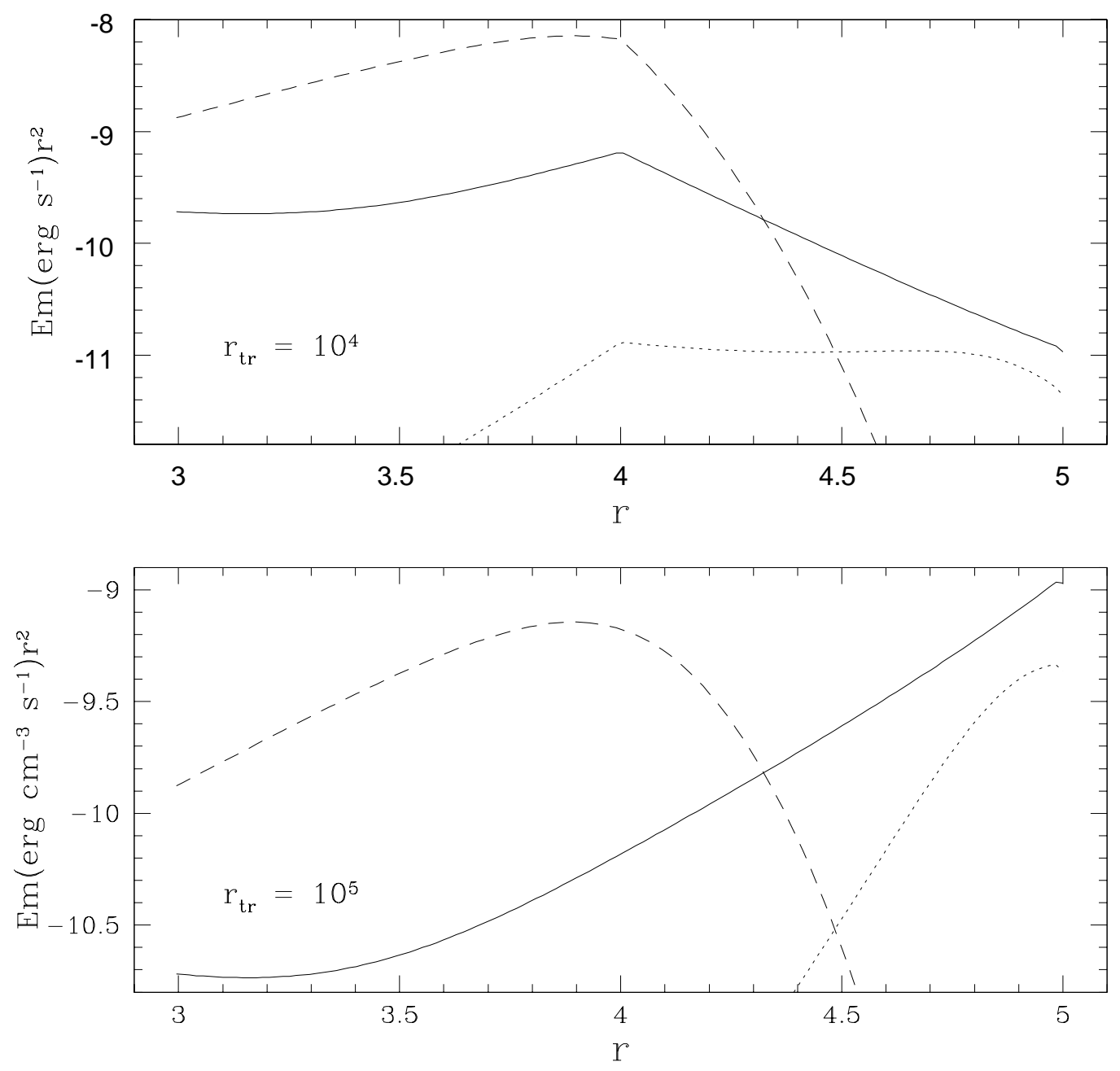

Fig. 9. - Emissivity in some of the strongest lines predicted for an ADAF in M87: Fe XXVI $\lambda 1.780$ (dashed line), O VIII $\lambda 18.97$ (solid line), and Si XIII $\lambda 6.648$ (dotted line). The two panels show the effect of varying $r_{\mathrm{tr}}$. The temperature profile is the same in both cases, but the density in the outer region is higher for larger $r_{\mathrm{tr}}$. This results in an enhancement of the total emission from the OVIII and Si XIII lines with respect to that from the Fe XXVI line. 